\title{
EFECTO DE LA QUEMA PRESCRIPTA DE UN PASTIZAL SOBRE EL BALANCE DE $\mathrm{CO}_{2}$
}

\author{
Bernardis, Aldo C. ${ }^{\text {; }}$ Fernandez, Juan A. ${ }^{\text {; }}$ Céspedes Flores, Flora ${ }^{1}$; \\ Goldfarb, María C. ${ }^{2}$; Casco, José F. ${ }^{2}$ \\ ${ }^{1}$ Facultad de Ciencias Agrarias - UNNE.qaaber@agr.unne.edu.ar \\ ${ }^{2}$ EEA INTA Corrientes
}

\section{RESUMEN}

El objetivo fue evaluar el efecto de la quema prescripta de un pastizal con predominio de gramíneas sobre la emisión y captura de $\mathrm{CO}_{2}$. El sitio no recibió quema por 25 años. Los tratamientos fueron: $\mathrm{T}_{1}=\sin$ quema; $\mathrm{T}_{2}=$ quema anual $\mathrm{y}_{3}=$ quema bianual. Se tomaron muestras de la biomasa aérea viva y muerta. Después de los eventos de quema se colectaron las cenizas. El contenido promedio de $\mathrm{C}$ del material vegetal fue del $45 \%$. La cantidad de $\mathrm{CO}_{2}$ liberado en promedio en forma anual para los eventos de quema fueron: 10.199 y $5.152 \mathrm{~kg} \mathrm{ha}^{-1}$ respectivamente para $\mathrm{T}_{2} \mathrm{y} \mathrm{T}_{3}$. En $\mathrm{T}_{1}$ se encontró una tendencia creciente en el contenido de carbono almacenado en la biomasa aérea viva y muerta, se consideró en equilibrio la emisión y la captura de $\mathrm{CO}_{2}$. En la quema anual se capturó $12.401 \mathrm{~kg}$ de $\mathrm{CO}_{2} \mathrm{ha}^{-1}$, la diferencia entre lo capturado y lo emitido fue de $2.202 \mathrm{~kg}$ de $\mathrm{CO}_{2} \mathrm{ha}^{-1}$. En la quema bianual se capturó $7.904 \mathrm{~kg}$ de $\mathrm{CO}_{2}$ en forma anual y por la quema se liberó $5.152 \mathrm{~kg} \mathrm{ha}^{-1}$, la diferencia entre lo capturado y lo emitido es de $2.752 \mathrm{~kg}$ de $\mathrm{CO}_{2}$. El $\mathrm{CO}_{2}$ liberado durante la quema es rápidamente capturado a través del proceso de fotosíntesis en el siguiente período de crecimiento vegetal. La captura de $\mathrm{CO}_{2}$ en los tratamientos que recibieron quema fue mayor a la emisión de $\mathrm{CO}_{2}$ durante el proceso de combustión de la biomasa.

PALABRAS CLAVE: carbono, pastoreo, efecto invernadero, manejo del fuego, cambio climático.

\footnotetext{
ABSTRACT

The objective was to evaluate the effect of the prescribed burning of grassland with predominance of grass on the emission and capture of $\mathrm{CO}_{2}$. The site did not receive burning for 25 years. The treatments were: $\mathrm{T}_{1}=$ without burning; $\mathrm{T}_{2}=$ annual burning and $\mathrm{T}_{3}=$ biennial burning. Samples were taken from the alive and dead aerial biomass. After the burning events the ashes were collected. The content average of Carbon of the vegetal material was of $45 \%$. The amount of $\mathrm{CO}_{2}$ released in av-
}

erage in annual form for the burning events was: 10199 and $5152 \mathrm{~kg} \mathrm{ha}^{-1}$ respectively for $\mathrm{T}_{2}$ and $\mathrm{T}_{3}$. In $\mathrm{T}_{1}$ was found an increasing tendency in the carbon content stored in the alive aerial biomass and dead, it was considered that the $\mathrm{CO}_{2}$ emissions and capture are balanced. In the annual burning 12401 $\mathrm{kg}$ of $\mathrm{CO}_{2} \mathrm{ha}^{-1}$ were captured, the difference between the captured and the emitted was of 2202 $\mathrm{kg}$ of $\mathrm{CO}_{2} \mathrm{ha}^{-1}$. In the biennial burning $7904 \mathrm{~kg}$ of $\mathrm{CO}_{2}$ were captured annually and in the burning $5152 \mathrm{~kg} \mathrm{ha}^{-1}$ were freed, the difference between the captured thing and the emitted thing is of 2752 $\mathrm{kg}$ of $\mathrm{CO}_{2}$. The $\mathrm{CO}_{2}$ released during the burning is quickly captured through process of photosynthesis in the following period of vegetal growth. The $\mathrm{CO}_{2}$ capture in the treatments that received burning was greater than the $\mathrm{CO}_{2}$ emission during the process of combustion of the biomass.

KEY WORDS: carbon, grazing, greenhouse effect, handling of the fire, climatic change.

\section{INTRODUCCIÓN}

La quema de biomasa aérea es una herramienta muy utilizada en las prácticas agropecuarias, permitiendo el control de malezas, eliminación de desechos agrícolas después de la cosecha. En los sistemas ganaderos para la eliminación del forraje que ha llegado a la madurez y que no es consumido por los animales por su baja palatabilidad y calidad (Hernández y Martinez, 2000). Estas prácticas de quemas, según la FAO (1996), puede ser considerado de dos maneras, uno es el fuego controlado o quema prescripta y el otro sin control. Cuando el fuego no es controlado puede llegar a ser destructivo; sin embargo cuando se controla se puede usar para quemar un área predeterminada con el objeto de alcanzar ciertos resultados deseados (Dentoni y Muñoz, 2001).

La combustión es la reacción química entre el oxígeno y un material oxidable, acompañada de desprendimiento de energía y que habitualmente se manifiesta por incandescencia o llama (Kunst, 
2001; BUN-CA, 2002). En la quema de pastizales el material oxidable está compuesto por vegetal vivo y muerto de diferentes especies. Los mismos están constituidos por agua, celulosa, hemicelulosa y lignina entre los compuestos más importantes de la biomasa aérea, el resto está formado por terpenos, taninos, resinas, aceites, ácidos orgánicos y minerales, componentes químicos que varían según el tipo de especies vegetales. Según López et al (1999), se puede asignar una fórmula química al combustible vegetal, que representa de manera aproximada la proporción de átomos de carbono, hidrógeno y oxígeno que podemos encontrar en diferentes pastizales. Esta fórmula es $\mathrm{C} 6 \mathrm{H} 9 \mathrm{O} 4$, y representa un 49,65 \% de C, $6.20 \% \mathrm{H}$ y 44,13 \% O. De esta manera, la ecuación general de la combustión de un combustible vegetal cuando es oxidado completamente quedaría de la siguiente manera:

$4 \mathrm{C} 6 \mathrm{H} 9 \mathrm{O} 4+25 \mathrm{O} 2 \rightarrow 24 \mathrm{CO} 2+18 \mathrm{H} 2 \mathrm{O}+11.6$ $\mathrm{x} 106$ joules

Por lo tanto, el calor de combustión promedio de un material vegetal es aproximadamente $20 \mathrm{~kJ}$ g-1 (4800 cal g-1).

Como producto de la combustión se han identificado más de 200 compuestos, entre los principales se encuentran $\mathrm{CO} 2$, agua, etano, propano, propileno, metil y etil-acetileno, ácidos orgánicos aldehídos y furanos. Durante el proceso de quema se genera un gran número de partículas, producidas en la pirólisis y combustión. La mayor parte de estas partículas son líquidas formadas por la condensación de vapores orgánicos y se presentan en formas esféricas (Lopez et al, 1999). Las partículas sólidas presentan formas diversas, con tamaños que varían entre $0.01 \mu \mathrm{m}$ y $5 \mu \mathrm{m}$, que forman el humo (Dentoni y Muñoz, 2001). Como producto de la quema queda depositado sobre el suelo el residuo de la combustión, denominado comúnmente cenizas. En realidad éste material está formado por materia orgánica parcialmente quemada y cenizas. Se logra obtener cenizas sin resto de material orgánico cuando la temperatura alcanza los $550^{\circ} \mathrm{C}$ durante 3 horas (A.O.A.C., 1997). En la práctica de la quema prescripta de pastizales esto no ocurre, si bien la temperatura puede superar los $550^{\circ} \mathrm{C}$, el tiempo que permanece es mucho menor y en consecuencia queda material vegetal sin quemarse. La combustión no es uniforme porque depende de varios factores: cantidad de material combustible, humedad del material, velocidad del viento, composición y características del material combustible (vegetales muertos, vegetales con cierto grado de descomposición, vegetales vivos).

El CO2 es el gas de efecto invernadero con mayor contribución real en el calentamiento global, siendo el gran responsable del fenómeno del Cambio Climático (IPCC, 1996; Slijepcevic, 2001). El sector agropecuario a través de sus actividades de producción contribuye directamente a la emisión de gases de efecto invernadero. En los sistemas silvopastoriles la fracción forestal contribuye en menor grado.

Cuando se quema materia seca combustible de biomasa vegetal, el dióxido de carbono liberado a la atmósfera es absorbido por las plantas y convertido en carbohidratos y tejidos a través del proceso de fotosíntesis, como parte del ciclo del carbono produciendo un balance natural (BUN-CA, 2002; Lapeyre et al., 2004). De hecho, la cantidad de carbono secuestrado puede ser mayor que el liberado durante la combustión, debido a que en muchas de las especies se quema solo una parte de la planta (biomasa aérea), las raíces, además de estabilizar los suelos, secuestran carbono en su regeneración año tras año.

Bajo la hipótesis de que la emisión del CO2 generado por la quema prescripta en forma bianual de los pastizales es menor a la captura de $\mathrm{CO} 2$ en la biomasa producida durante el año, se realizó esta experiencia.

\section{OBJETIVOS}

El objetivo fue evaluar el efecto de la quema prescripta de un pastizal sobre la emisión y captura de $\mathrm{CO}_{2}$ en el noroeste de la provincia de Corrientes.

\section{MATERIALES Y MÉTODOS}

En el año 1997 se estableció un ensayo sobre un pastizal situado en la Estación Experimental Agropecuaria - INTA Corrientes, $\left(27^{\circ} 39^{\prime}\right.$ S y $58^{\circ} 46^{\prime} \mathrm{W}$ y 57 m.s.n.m.) ubicada en la región occidental de la Provincia, Departamento de Empedrado, dentro del área ecológica del Albardón Río Paraná. El clima está clasificado como subtropical isohigro sin estación seca (Pappadakis, 1975). La temperatura media anual es de $21^{\circ} \mathrm{C}$. El número de horas frío anuales (temperaturas $<10^{\circ} \mathrm{C}$ ) promedian las 250 horas, variando de 100 a 350 horas según los años. Las heladas ocurren entre los meses de Mayo y Octubre, registrándose en promedio 1,4 heladas meteorológicas y 10 agronómicas. Las precipitaciones oscilan entre 798 a $2.130 \mathrm{~mm}$, con un promedio anual de 1.308,2 mm (Serie 1960-2000). La humedad relativa media varía entre 70 \% y $75 \%$ (INTA, 2007). El suelo pertenece a la serie Valencia, que se caracteriza por ser muy ácido, de baja fertilidad, drenaje imperfecto, permeabilidad y escurrimiento lento con una profundidad efectiva de raíces de $73 \mathrm{~cm}$. El índice de productividad es 5 y la capacidad de uso es VIw. El uso actual es para ganadería extensiva y en menor escala para el cultivo del arroz (Escobar et al., 1996).

El pastizal estudiado corresponde a una comunidad vegetal del tipo albardón con una productividad promedio de $6.000 \mathrm{~kg} \mathrm{ha}^{-1}$ año ${ }^{-1}$ de biomasa seca disponible, con predominio de Andropogon 
lateralis (paja colorada) y Sorghastrum setosum (paja amarilla). En base a los resultados de trabajos preliminares realizados por Casco (1993), se estudiaron tres frecuencias de quema, con los siguientes tratamientos; $\mathrm{T}_{1}=\sin$ quema; $\mathrm{T}_{2}=$ quema anual y $\mathrm{T}_{3}=$ quema bianual. El lote testigo $\left(\mathrm{T}_{1}\right)$, corresponde a una parte de la superficie del ensayo que no ha recibido ningún evento de quema por más de 25 años. Establecidas las parcelas, el 20 de agosto 1997, para uniformar la vegetación se desmalezó el tratamiento sin quema $\left(\mathrm{T}_{1}\right)$ con una máquina desmalezadora mecánica, con una altura de corte de aproximadamente $15 \mathrm{~cm}$. En la misma fecha los tratamientos $\mathrm{T}_{2}$ y $\mathrm{T}_{3}$ fueron quemados. El tratamiento $\mathrm{T}_{2}$ se quemó en forma anual desde 1997 hasta 2005 (nueve eventos de quema). El tratamiento $\mathrm{T}_{3}$ se quemó cada 2 años hasta 2005 (cinco eventos de quema). Todos los tratamientos fueron pastoreados después de efectuar los cortes de evaluación con ganado vacuno, se utilizaron animales y régimen de pastoreo del Sistema Experimental de Cría Vacuna (SECV) de la EEA, INTA Corrientes, con una carga animal promedio de 0,50 unidades ganadera ha-1 ${ }^{-1}$ cuyo criterio de uso fue mantener hasta una disponibilidad de $2500 \mathrm{~kg}$ $\mathrm{ha}^{-1}$ de biomasa acumulada de forraje remanente (Arias Mañotti et al., 2003).

El diseño experimental fue en bloques completos al azar con cuatro repeticiones. Las parcelas experimentales tenían una superficie de $2000 \mathrm{~m} 2$ separadas por contrafuegos de $4 \mathrm{~m}$ de ancho. Las quemas se realizaron al final de cada periodo invernal. De cada unidad experimental se tomaron muestras de material vegetal en las distintas estaciones del año. En cada estación del año y antes del evento de quema se realizó un muestreo de la parte aérea de la vegetación para determinar la cantidad de materia seca combustible de biomasa aérea. El material vegetal fue tomado de cuatro repeticiones mediante cortes al ras del suelo y recolección del material seco depositado en la superficie del suelo, de un cuadro de 0,25 m2. El material cosechado se secó en estufa con circulación de aire forzado a $65{ }^{\circ} \mathrm{C}$ hasta peso constante. Se determinó el porcentaje de humedad y disponibilidad de biomasa seca.

Antes de realizar los eventos de quema se monitoreó las condiciones ambientales y se comparó con el protocolo de quema, con los siguientes parámetros: Velocidad del viento: Entre 9 a 24 km h-1. Humedad relativa del aire: Entre 25 y $40 \%$. Temperatura del aire: Permaneció entre los 10 y $33^{\circ} \mathrm{C}$.

No se realizó la quema con vientos de velocidad superior a $24 \mathrm{~km} \mathrm{~h}-1$ y temperatura del aire inferiores a $\operatorname{los} 10^{\circ} \mathrm{C}$. La temperatura del aire ideal para la mayoría de las quemas es entre 20 y $25^{\circ} \mathrm{C}$ (Moscovich y Lacorte, 2003)

Cuando las condiciones ambientales fueron propicias según las referencias se quemaron las par- celas experimentales correspondientes en el mes de agosto (invierno). Para producir la ignición se emplearon antorchas y el fuego fue en sentido contrario al viento predominante. Durante los eventos de quemas se midió la temperatura en la superficie del suelo y a una profundidad de 0,5 y $2 \mathrm{~cm}$ y la temperatura de la llama a una altura de $50 \mathrm{~cm}$ del suelo, mediante el uso de lápices indicadores de temperaturas (Bernardis, 2008) . Se determinó la velocidad de propagación del fuego (Dentoni y Muñoz, 2001).

Después de realizada la quema se recolectaron las cenizas de una superficie de $0,50 \times 0,50 \mathrm{~m}$. Se pesaron y determinaron los $\mathrm{kg}$ ha-1 de cenizas producidas en los diferentes tratamientos. Para el tratamiento T3 se estimó la masa de cenizas en forma anual (masa de cenizas en cada evento dividido dos). Una parte de las cenizas colectadas en las parcelas experimentales fue incinerada en una mufla a $550^{\circ} \mathrm{C}$ durante 3 horas para determinar el contenido real de cenizas, por el método de la A.O.A.C. (1997). Por diferencia entre la materia seca y el contenido de cenizas se determinó la cantidad de materia orgánica. El carbono del material vegetal es variable y para realizar una estimación se consideró para los pastizales un contenido de carbono del 45 \% (Álvarez, 2006).

El cálculo de la emisión y captura de CO2 por el pastizal, se determinó por medio de aplicaciones matemáticas sencillas, partiendo del peso del carbono presente en la biomasa seca y multiplicándolo por la relación existente entre el peso molecular del CO2 y el peso atómico del carbono (12); es decir que por cada $\mathrm{Kg}$ de carbono encontrado en la materia seca de la biomasa del pastizal cuando se quema completamente emite $3,67 \mathrm{Kg}$ de $\mathrm{CO} 2$. El CO2 liberado durante la quema es capturado a través del proceso de fotosíntesis en el siguiente período de crecimiento vegetal. Por cada kg de materia seca producido se ha retirado de la atmósfera $1,65 \mathrm{~kg}$ de CO2 y se ha liberado 1,20 kg de O2. De esta forma podemos comparar las emisiones de $\mathrm{CO} 2$ en forma anual. No se consideró la emisión de CO2 producida por la respiración del pastizal.

Para el cálculo de la cantidad de CO2 liberado en forma anual se consideró para la quema bianual (T2) la cantidad de CO2 liberado dividido 2 (se quema cada 2 años).

Con los datos se realizó un ANOVA y las diferencias entre medias se probaron con test de Tukey $(\mathrm{p} \leq 0,05)$.

\section{RESULTADOS Y DISCUSIÓN}

La cantidad de materia seca combustible de biomasa aérea acumulada promedio de 6 años, para los tratamientos y estaciones del año se presentan en la Figura 1. En el momento de la quema (invierno) se registraron valores promedio de: 7.254, $7.516 \mathrm{y}$ 
Cuadro 1. Valores registrados de parámetros ambientales y del material combustible durante los eventos de quema de pastizales.

\begin{tabular}{lcc}
\hline \multicolumn{1}{c}{ Parámetros } & \multicolumn{2}{c}{ Rango de variación } \\
& Mínimo & Máximo \\
\hline Temperatura en la superficie del suelo & $280^{\circ} \mathrm{C}$ & $350^{\circ} \mathrm{C}$ \\
Temperatura del suelo a 0.5 cm de profundidad & $149^{\circ} \mathrm{C}$ & $186^{\circ} \mathrm{C}$ \\
Temperatura del suelo a 2 cm de profundidad & $59^{\circ} \mathrm{C}$ & $70^{\circ} \mathrm{C}$ \\
Temperatura de la llama a 50 cm del suelo & $350^{\circ} \mathrm{C}$ & $580^{\circ} \mathrm{C}$ \\
Humedad del material combustible & $41 \%$ & $58 \%$ \\
Velocidad del viento & $9 \mathrm{~km} \mathrm{~h}^{-1}$ & $22 \mathrm{~km} \mathrm{~h}^{-1}$ \\
Humedad relativa del aire & $40 \%$ & $77 \%$ \\
Velocidad de propagación del fuego & $0,3 \mathrm{~m} \mathrm{~min}^{-1}$ & $1,4 \mathrm{~m} \mathrm{~min}^{-1}$ \\
\hline
\end{tabular}

Cuadro 2: Dióxido de carbono $\left(\mathrm{CO}_{2}\right)$, liberado, capturado, balance anual y energía generada en los eventos de quema de pastizales con manejo del fuego en forma anual y bianual. Datos promedio de 6 años de ensayo.

\begin{tabular}{lcc}
\hline \multicolumn{1}{c}{ Componentes } & \multicolumn{2}{c}{ Tratamientos } \\
\cline { 2 - 3 } & Quema anual & Quema bianual \\
\hline $\mathrm{Kg}$ de $\mathrm{CO}_{2}$ liberado ha-1 año & $10.199 \mathrm{a}$ & $5.152 \mathrm{~b}$ \\
$\mathrm{Kg}$ de $\mathrm{CO}_{2}$ capturado ha-1 año & $12.401 \mathrm{a}$ & $7.904 \mathrm{~b}$ \\
Balance de $\mathrm{CO}_{2}$ ha-1 año & $2.202 \mathrm{~b}$ & $2.752 \mathrm{a}$ \\
Energía liberada kJ ha-1 año & $111.168 \mathrm{a}$ & $56.151 \mathrm{~b}$ \\
\hline
\end{tabular}

Letras distintas en la fila indican diferencias significativas (Tukey, $\mathrm{p} \leq 0,05$ ).

$7.588 \mathrm{~kg} \mathrm{ha}^{-1}$ respectivamente para los tratamientos $\mathrm{T}_{1}, \mathrm{~T}_{2} \mathrm{y} \mathrm{T}_{3}$.

En el tratamiento con quema bianual, la materia seca de biomasa aérea fue mayor con diferencias significativas $(p<0,05)$ en los muestreos de primavera y verano respecto al testigo. En el resto de las estaciones, las diferencias no fueron significativas $(\mathrm{p} \geq 0,05)$. En el testigo, la materia seca acumulada fue menor que el resto de los tratamientos en primavera y verano. En primavera se registró la mayor acumulación para el tratamiento quema bianual, con $9.500 \mathrm{~kg} \mathrm{ha}^{-1}$, incrementando $152 \%$ y 88 $\%$, respecto del testigo y el tratamiento de quema anual respectivamente. La acumulación de biomasa del tratamiento con quema anual fue de $5.055 \mathrm{~kg}$ ha $^{-1}$, sin diferir significativamente del testigo.

Durante los eventos de quema del pastizal las condiciones ambientales y del material combustible fueron monitoreadas y los resultados obtenidos con sus valores mínimos y máximos se presentan en el Cuadro 1.

Los registros de temperatura a nivel de suelo obtenidos en esta experiencia, fueron similares a los rangos de temperatura presentado por Vargas et a.l, (2004) en trabajos realizado en Perú, quienes observaron que la quema controlada no incrementa sustancialmente la temperatura, y esta observación coincide con lo evaluado por Chancayauri (1999) y Argote (1999) en suelos de otras regiones de pastizales de puna. De acuerdo a estas observaciones de poca variación de la temperatura y en corto tiempo se puede inferir que no tendría efecto negativo sobre la microflora del suelo. El porcentaje de materia orgánica contenida en las cenizas re-
Figura 1. Acumulación de materia seca de biomasa aérea en distintas estaciones del año con pastoreo. Letras distintas indican diferencias significativas $(\mathrm{p} \leq 0,05)$.

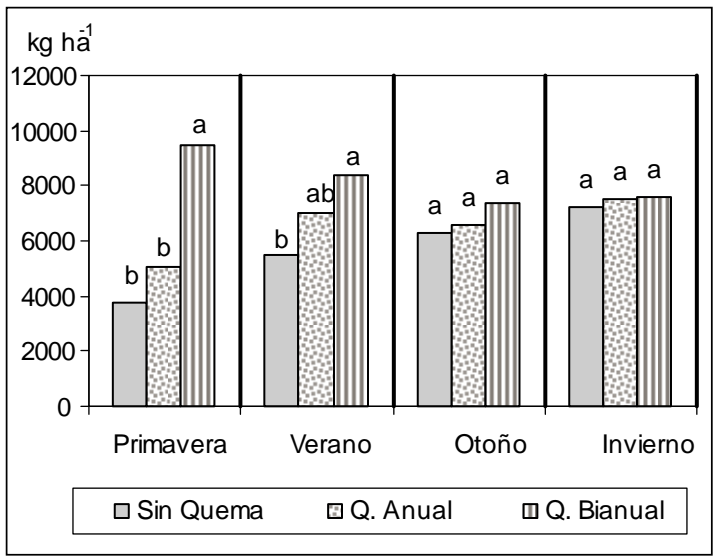

manentes, en promedio fueron $17,83 \%$ y $17,78 \%$, respectivamente para los tratamientos $\mathrm{T}_{2} \mathrm{y} \mathrm{T}_{3}$. La cantidad de material vegetal que se quemó fue de 6.176 y $6.239 \mathrm{~kg} \mathrm{ha}^{-1}$ para los tratamientos $\mathrm{T}_{2} \mathrm{y} \mathrm{T}_{3}$. Teniendo como base fórmula química del material vegetal dada por Lopez et al. (1999) y para poder incorporar la fracción mineral se propone la siguiente ecuación para representar el material combustible:

$$
\begin{gathered}
4 \mathrm{C}_{6} \mathrm{H}_{9} \mathrm{O}_{4}(\mathrm{M})+24 \mathrm{O}_{2} \rightarrow 24 \mathrm{CO}_{2}+18 \mathrm{H}_{2} \mathrm{O}+\mathrm{N}_{2} \mathrm{O}+ \\
\mathrm{SO}_{2}+\text { Cenizas }+10.67 \times 10^{-6} \mathrm{~J}
\end{gathered}
$$

Donde $\mathrm{M}$ representa a los minerales presentes en el material vegetal.

En base a la ecuación presentada, la energía liberada en la combustión por gramo de materia seca de biomasa aérea es de aproximadamente $18 \mathrm{~kJ}$. 
Considerando esta relación, el carbono representa un $45.18 \%$, el hidrógeno un $5.64 \%$ y el oxígeno un $40.16 \%$. Levine et al (1995), Álvarez (2006) consideran que la biomasa aérea de los vegetales contiene aproximadamente un $45 \%$ en peso de carbono.

La cantidad de $\mathrm{CO}_{2}$ liberado en promedio en forma anual para los eventos de quema se presenta en el Cuadro 2.

En la quema anual, se genera $10.199 \mathrm{~kg}$ de $\mathrm{CO}_{2} \mathrm{ha}^{-1}$ y en el período de crecimiento del pastizal (desde la quema hasta el invierno) captura $12.401 \mathrm{~kg}$ de $\mathrm{CO}_{2}$ ha $^{-1}$. La emisión de CO2 en esta experiencia con la quema anual es superior a lo obtenido por Barbosa y Fearnside (2005), quienes obtuvieron 4928,55 kg de $\mathrm{CO}_{2}$ ha $^{-1}$ emitidos a la atmósfera después de la quema de pastizales de sabanas y estepas en la Amazonia brasilera. Sin embargo, Kauffman et al. (1994) registraron en ecosistemas similares en el centro de Brasil, una emisión a la atmósfera de $11.124,7 \mathrm{~kg}$ de $\mathrm{CO}_{2} \mathrm{ha}^{-1}$. La diferencia entre lo capturado y lo emitido es de $2.202 \mathrm{~kg}$ de $\mathrm{CO}_{2} \mathrm{ha}^{-1}$. En la quema bianual produce en primavera un incremento en la disponibilidad de biomasa aérea de $4.940 \mathrm{~kg} \mathrm{ha}^{-1}$, respecto de la quema anual, esto significa que se ha capturado $7.904 \mathrm{~kg}$ de $\mathrm{CO}_{2}$ en forma anual de la atmósfera. Por efecto de la quema bianual libera únicamente $5.152 \mathrm{~kg} \mathrm{ha}^{-1}$ la diferencia entre lo capturado y lo emitido es de $2.752 \mathrm{~kg}$ de $\mathrm{CO}_{2}$.

\section{CONCLUSIONES}

El $\mathrm{CO}_{2}$ emitido por efecto de la quema anual o bianual de pastizales es inferior al capturado por los mismos a lo largo del año.

La quema prescripta en forma anual o bianual produce un balance positivo por capturar mayor cantidad de $\mathrm{CO}_{2}$ que lo emitido durante este evento.

\section{BIBLIOGRAFÍA}

A.O.A.C. Official Methods of Analysis .1997. 16th Edition 3rd Revision. Ahlgren, I.F. y Ahlgren, C.E. (1960). Ecological effects of forest fires. Bot. Rev., Vol. 26: 483-533.

Álvarez, R., Steinbach, H. S., Lavado, R. S. y Gutiérrez Boem, F. H. 2006. Materia Orgánica. Valor Agronómico y Dinámica en Suelos Pampeanos. Editorial Facultad Agronomía. Universidad de Buenos Aires. $1^{\mathrm{a}}$ ed. 256 p.

Argote, G. 1999. Respuesta de la producción primaria, composición floristica y valor nutritivo de pastizales dominados por Calamagrostis antoniana y Festuca dolicophylla a l.a quema prescripta frontal. Especialidad de Producción Animal. Tesis para optar al grado de Magister Scientiae. Universidad Nacional Agrária La Molina. Lima, Perú.
Arias Mañotti, AA, Goldfarb, M.C., Slobodzian, A. Gimenez, LI, y Nuñez, F. 2003. Variación interanual de la eficiencia de cosecha en pastizales del N.O. de Corrientes. Revista Argentina de Producción Animal Vol 23 Supl 1: 139-140.

Barbosa, R. I. and Philip, M. F. 2005. Aboveground biomass and the fate of carbon after burning in the savannas of Roraima, Brazilian Amazonia. Forest Ecology and Management. Vol. 216: 295-316.

Bernardis, A.C. 2008. Evaluación del Impacto ambiental de quemas prescriptas en pastizales en el N.O. de Corrientes. Tesis presentada para optar al grado de Magíster en Ingeniería en Calidad. Universidad Tecnológica Nacional. Facultad Regional Resistencia. Maestría en Ingeniería en Calidad. 53 p.

BUN-CA (Biomass Users Network). 2002. Manuales sobre energía renovable: Biomasa. 1 ed. -San José, C.R. 42 p. il.

Casco, J.F. 1993. El uso del fuego en los pastizales del N.O. de la provincia de Corrientes. Biología y manejo de fuego en ecosistemas naturales y modificados. Memoria del Seminario Taller. 2, 3 y 4 de Junio de 1993. EEA INTA Santiago del Estero. pp. 139-140.

Chancayauri, R. 1999. Dinámica de la producción primaria, composición florística y valor nutritivo de pastizales dominados por Dolychophylla y Calamagrostis vicunarum en respuesta a la quema prescripta frontal. Tesis para optar por el graqdo de Mgister Scientiae de la especialidad de Producción Animal. UNALM.

Dentoni, M.C. y Muñoz M .2001. Glosario de Términos Relacionados con el Manejo del Fuego. Plan Nacional de Manejo del Fuego Secretaría de Desarrollo Sustentable y Política Ambiental. República Argentina, 30 p.

Escobar, E.; Ligier, D.; Melgar, R.; Matteio, H. y Vallejos, O. 1996. Mapa de Suelos de la Provincia de Corrientes.1:500.000. INTA: EEA Corrientes.432 p.

FAO. 1996. Principios de Manejo de Praderas Naturales. Instituto Nacional de Tecnología Agropecuaria - INTA Argentina - y Oficina Regional de la FAO para América Latina y el Caribe. $2^{\text {da }}$ Ed. Santiago, Chile. 273 p.

Hernández, M.C. y Martinez, A. 2000. Quemas o incendios. Efectos sobre los recursos naturales. ubdireccion de gestion ambiental http://www. corpochivor.gov.co/cosmos 0100eslrn.htm Cosmos Año 4 / No 13.

INTA. 2007. Instituto Nacional de Tecnología Agropecuaria. EEA Corrientes. http://www. inta.gov.ar/corrientes/index.htm 08-07-07.

IPCC 1996. Intergovernmental Panel on Climate Change. Chapter 5: Land Use Change \& Forestry. Greenhouse Gas Inventory Reference 
Manual. IPCC Guidelines for National Greenhouse Gas Inventories, Revised Version. London, Vol. 3: 57 p.

Kauffman, J.; Cummings, D. and Ward, D. 1994. Relationships of fire, biomass and nutrients dynamics along a vegetations gradient in the Brazilians Cerrado. Journal Ecology. Vol. 82: 519-531.

Kunst C. 2001. El fuego como herramienta de manejo del pastizal natural. INTA EEA Santiago del Estero, Argentina. www.producción-animal.com.ar 6 p.

Lapeyre, T.; J. Alegre y L. Arévalo. 2004. Determinación de las reservas de carbono de la biomasa aérea, en diferentes sistemas de uso de la tierra en San Martín, Peru. Ecología Aplicada, Vol. 3: 1-2.

Levine J. S.; Cofer W. R.; Cahoon D. R. y Winstead E. L. 1995. Biomass burning: a driver for global change. Environmental science \& technology (Environ. sci. technol.) ISSN 0013936X CODEN ESTHAG Environmental science and technology. Vol. 29, No3: 120-125.
López, F.; J. Meléndez y A.J. de Castro.1999. Los incendios forestales. Aspectos medioambientales y tecnológicos I. Revista Mensual de Gestión Ambiental, $\mathrm{N}^{\mathrm{0}}$ 12: 27-36.

Moscovich, F. y Lacorte, S. 2003. Fuego prescripto en el manejo de pastizales. La Palanca Vol. 5: $3-9$.

Pappadakis, J. 1975. Las áreas subtropicales y sus posibilidades. Delimitación y Regiones ecológicas de Argentina subtropical. Primeras Jornadas Argentinas y Formoseñas de las Áreas Subtropicales Argentinas. AACREA y CREA Formosa. pp 8-10.

Slijepcevic, A. 2001. Loss of carbon during controlled regeneration burns in Eucalyptus oblicua forest. Tasforest, Vol. 13, No 2: 281-290.

Vargas, D.; Guerra, R. y Morales, J. 2004. Efecto de una quema controlada sobre la población microbiana en suelos con pasturas en la Sais Tupac Amaru - Junin, Perú. Ecologia aplicada, Vol. 3. 